\title{
Completely Integrable Models of Non-linear Optics
}

\author{
Andrey I. Maimistov \\ Department of Solid State Physics, Moscow Engineering Physics Institute, \\ Moscow, 115409, Russia \\ electronic address: maimistov@pico.mephi.ru
}

\begin{abstract}
The models of the non-linear optics in which solitons were appeared are considered. These models are of paramount importance in studies of nonlinear wave phenomena. The classical examples of phenomena of this kind are the self-focusing, self-induced transparency, and parametric interaction of three waves. At the present time there are a number of the theories based on completely integrable systems of equations, which are both generations of the original known models and new ones. The modified Korteweg-de Vries equation, the non- linear Schrodinger equation, the derivative nonlinear Schrodinger equation, Sine-Gordon equation, the reduced MaxwellBloch equation, Hirota equation, the principal chiral field equations, and the equations of massive Thirring model are gradually putting together a list of soliton equations, which are usually to be found in non-linear optics theory.
\end{abstract}

Keywords: solitons, self-induced transparency, non-linear fibres, wave interaction PACS number: 42.65. 


\section{Introduction}

A non-linear wave is one of the fundamental objects of nature. They are inherent to aerodynamics and hydrodynamics, solid state physics and plasma physics, optics and field theory, chemistry reaction kinetics and population dynamics, nuclear physics and gravity. All non-linear waves can be divided into two parts: dispersive waves and dissipative ones. The history of investigation of these waves has been lasting about two centuries. In 1834 J.S. Russell discovered the extraordinary type of waves without the dispersive broadening. In 1965 N.J. Zabusky and M. D. Kruskal found that the Korteweg-de Vries equation has solutions of the solitary wave. This solitary wave demonstrates the particle-like properties, i.e., stability under propagation and the elastic interaction under collision to one another. For these reasons these solitary waves were named solitons. In succeeding years there has been a great deal of progress in understanding of soliton nature. Now solitons have become the primary components in many important problems of non-linear wave dynamics. It should be noted that non-linear optics is the field, where all soliton features are exhibited to a great extent.

The self-focusing of light beams and self-induced transparency phenomenon set the bright example of an important role of solitons in non-linear optics.

The non-linear wave propagation in non-resonant medium also occupies an important place in non-linear optics. As c consequence of modulation instability, continuous wave transforms to number of single pulses. These pulses can under certain conditions evaluate into solitons. Nowadays the optical solitons propagation in fibres has attracted considerable attention.

The other classical problem of non-linear optics is the parametric interaction of waves. The harmonic generation, stimulated by both Raman and Brillouin scattering, parametric amplifications, sum-frequency mixing, and four-wave mixing have been the subject of many investigations in this field. The three-wave interaction process is of particular interest because it gives us a new instance of the application of the soliton occurrence in non-linear system without dispersion.

There are cases of the Hamiltonian systems when the canonical transformation makes the equations of motion trivially integrable after the conversion to new variables. Then they say that Hamiltonian system admits the action-angle variables. If the action-angle variables exist, then the Hamiltonian system is completely integrable. There is powerful tool to investigate the integrable systems. It is Inverse Scattering Transform (IST) introduced by 
Gardner, Greene, Kruskal and Miura (1967). The implementation of IST in non-linear optics results in new examples of the optical soliton phenomena. Moreover, this method leads to new analytical approaches for near integrable systems.

\section{Classical examples}

Let us consider three phenomena mentioned above for that theories have been based on completely integrable equations.

\subsection{Self-induced transparency}

A self-induced transparency (SIT) phenomenon consists in the propagation of a powerful ultra-short pulse (USP) of light through a resonance medium without the distortion and energy loss of this pulse [1, 2, 3, 4]. This phenomenon is characterised by the continuous absorption and re-emission of electromagnetic radiation by resonant atoms of medium in such a manner that steady-state optical pulse propagates. In the ideal case the energy dissipation of the USP is invisible and the state of the resonant medium is not varying. In this means the medium is transparent. The group velocity of such steady-state pulse, called $2 \pi$-pulse or soliton of SIT, is less than the phase speed of light in a medium. The group velocity depends on a $2 \pi$-pulse duration: the shorter is the duration, the higher is its speed [2, 3, 4, 5]. When two pulses of the different velocities spread in the medium, the second pulse may overtake the first and a collision will take place. After the collision, the solitons keep their shape and velocity (but in general all other parameters of solitons may alter). This fundamental property of the SIT solitons has been studied many times both theoretically and experimentally [3, 6, 7].

From the mathematical point of view this property is a consequence of the complete integrability of the reduced Maxwell-Bloch equations, describing the SIT in the two-level media with non-degenerated levels [8] - [13]. The $2 \pi$-pulses answer the single- soliton solutions of these equations, and the process of "collision" reflects the evolution of the double-soliton solution - its asymptotically transformation into a pair of solitons under certain conditions (see, for example, [6, 9], and [14] - 15]).

The simplest theory describing the self-induced transparency phenomenon was developed by McCall and Hahn [1, 2]. In general, the theory of the in- 
teraction of radiation with an ensemble of two-level atoms is based on the Bloch equations for atoms and the Maxwell equations for the classical electromagnetic field. In an isotropic dielectric the set of Maxwell equations reduced to one equation for the electric field $\vec{E}=\vec{l} E$. For a plane wave with constant polarisation vector $\vec{l}$ one can obtain the following system of total Maxwell-Bloch (MB) equations

$$
\begin{gathered}
\frac{\partial^{2} E}{\partial z^{2}}-\frac{1}{c^{2}} \frac{\partial^{2} E}{\partial t^{2}}=\frac{4 \pi n_{A} d}{c^{2}}\left\langle\frac{\partial^{2} r_{1}}{\partial t^{2}}\right\rangle \\
\frac{\partial r_{1}}{\partial t}=-\omega_{a} r_{2}, \quad \frac{\partial r_{2}}{\partial t}=\omega_{a} r_{1}+\frac{2 d}{\hbar} E r_{3}, \quad \frac{\partial r_{3}}{\partial t}=-\frac{2 d}{\hbar} E r_{2}
\end{gathered}
$$

where $d$ is the projection of a matrix element of the dipole operator on the direction of $\vec{l}, n_{A}$ is the concentration of resonant atoms. It should be noted that the components of Bloch vector $r_{1}, r_{2}$, and $r_{3}$ depend on the atomic resonance frequency $\omega_{a}$. Hereafter the angular brackets represent summation over all the atoms characterised by the frequency $\omega_{a}$.

The Bloch equations contain products of the field $E$ and the polarisations $r_{2}$ and $r_{3}$ responsible for interference between the opposite propagated waves. It has been shown [16, 17], however, that if the density of resonant atoms is small enough to make the parameter $4 \pi n_{A} d^{2} / \hbar \omega_{a}$ less than unity, interference may be neglected. It was found that for a typical value of $d \sim$ 1 Debye, $\omega_{a} \sim 10^{15} \mathrm{~s}^{-1}$ and $n_{A} \ll 10^{23} \mathrm{~cm}^{-3}$ one may not take into account the backward wave generation by a forward running pulse. Thus, the MB equations convert into the simple reduced Maxwell-Bloch (RMB) system of equations:

$$
\begin{gathered}
\frac{\partial E}{\partial z}+\frac{1}{c} \frac{\partial E}{\partial t}=-\frac{2 \pi n_{A} d}{c}\left\langle\frac{\partial r_{1}}{\partial t}\right\rangle \\
\frac{\partial r_{1}}{\partial t}=-\omega_{a} r_{2}, \quad \frac{\partial r_{2}}{\partial t}=\omega_{a} r_{1}+\frac{2 d}{\hbar} E r_{3}, \quad \frac{\partial r_{3}}{\partial t}=-\frac{2 d}{\hbar} E r_{2} .
\end{gathered}
$$

It should be emphasised that in both the MB and RMB equations symbol $E$ denotes the real value of the electric field strength. However, every so often the electromagnetic wave can be represented as a quasi-monochromatic one

$$
E(z, t)=2 A(z, t) \cos \left[k_{0} z-\omega_{0} t+\varphi(z, t)\right]=\mathcal{E}(z, t) \exp \left[i\left(k_{0} z-\omega_{0} t\right)\right]
$$


where $\omega_{0}$ is the radiation frequency, $k_{0}$ is the wave number, and the real envelope $A(z, t)$ and the phase $\varphi(z, t)$ are slowly varying functions of $z$ and $t$. This is an approximation which means that the envelope and the phase obey the inequalities

$$
|\partial A / \partial t| \ll \omega_{0}|A|,|\partial A / \partial z| \ll k_{0}|A|,|\partial \varphi / \partial t| \ll \omega_{0}|\varphi|,|\partial \varphi / \partial z| \ll k_{0}|\varphi| .
$$

Besides, the envelope amplitudes are usually so weak that Rabi frequency $(\max |d A / \hbar|)$ turns out to be much less than the resonance transition frequency. The resulting system of equations can be represented as

$$
\begin{gathered}
\frac{\partial q}{\partial z}+\frac{1}{c} \frac{\partial q}{\partial t}=-\alpha^{\prime}\langle P\rangle, \quad q\left(\frac{\partial \varphi}{\partial z}+\frac{1}{c} \frac{\partial \varphi}{\partial t}\right)=\alpha^{\prime}\langle Q\rangle \\
\frac{\partial Q}{\partial t}=\left(\Delta \omega+\frac{\partial \varphi}{\partial t}\right) P, \quad \frac{\partial P}{\partial t}=-\left(\Delta \omega+\frac{\partial \varphi}{\partial t}\right) Q+q R, \quad \frac{\partial R}{\partial t}=-q P .
\end{gathered}
$$

where $\Delta \omega=\left(\omega_{a}-\omega_{0}\right), \alpha^{\prime}=2 \pi \omega_{0} n_{A} d^{2} / \hbar c, q=d A / \hbar$ is a normalised slowly varying pulse envelope and $P, Q$ and $R$ are connected with initial Bloch vector components by the relations

$$
\begin{aligned}
& r_{1}=P(z, t) \sin \left[k_{0} z-\omega_{0} t+\varphi(z, t)\right]+Q(z, t) \cos \left[k_{0} z-\omega_{0} t+\varphi(z, t)\right], \\
& r_{3}=-R(z, t)
\end{aligned}
$$

To describe the SIT phenomenon McCall and Hahn used the system (7) and (8). Furthermore, if we confine the analysis to situations when the input optical pulse does not carry any phase modulation, i.e. $\partial \varphi / \partial z=\partial \varphi / \partial t=0$ at $\mathrm{z}=0$ and the form factor of the inhomogeneous line is a symmetrical function of frequency detuning $\Delta \omega$, then equations (17) and (8) yield $\partial \varphi / \partial z=$ $\partial \varphi / \partial t=0$ at any $z$ and $t$. In this case equations (7) and (8) reduce to the system of SIT equations

$$
\begin{gathered}
\frac{\partial q}{\partial z}+\frac{1}{c} \frac{\partial q}{\partial t}=-\alpha^{\prime}\langle P\rangle, \\
\frac{\partial Q}{\partial t}=\Delta \omega P, \quad \frac{\partial P}{\partial t}=-\Delta \omega Q+q R, \quad \frac{\partial R}{\partial t}=-q P .
\end{gathered}
$$


If an absorption line is homogeneously broadened and exact resonance condition holds, then the SIT equations reduce to the well-known Sine-Gordon equation

$$
\frac{\partial^{2} u}{\partial \tau \partial \zeta}+\sin u=0
$$

where $\tau=(t-z / c), \zeta=\alpha^{\prime} z$, and $q=\partial u / \partial \tau$.

These equations (i.e., (3 - 4), (7) - 8), and (?? - ??)) may be represented as the condition of the integrability of some linear equations that provides to the solution of these equations be the IST method. If we assume, that before the arrival of an ultra-short pulse all two-level atoms are in the ground state and after the passing of the USP all atoms recover in the initial states, then boundary conditions are

$$
\lim _{|\tau| \rightarrow \infty} R\left(\tau, \zeta ; \omega_{a}\right)=-1, \quad \lim _{|\tau| \rightarrow \infty} r_{1,2}\left(\tau, \zeta ; \omega_{a}\right)=0
$$

Both RMB-equations and SIT-equations with these boundary conditions can be solved by the IST method in a regular way [8] - [15]. In general case one obtain the $N$-soliton solution of these equations. This solution represents $L_{1}$ single solitons and $L_{2}$ breathers (so that $N=L_{1}+2 L_{2}$ ). Breather (or bion soliton-antisoliton bounded state) is an extremely stable solitary wave with internal oscillations. It has the same collision stability as ordinary solitons in both the bion-bion and the bion-soliton collisions. It is worth to note that the Sine-Gordon equation has the same soliton and breather solution.

It should be note that one-soliton solution of the RMB equations corresponds the USP without carrier wave and represents a unipolar spike of electromagnetic radiation. Sometimes these pulses are named the video pulses. Two-soliton solution of the RMB-equations describes the collision of two video pulses in the same fashion as it was done for two soliton solution of SIT equations by McCall-Hahn. However, the breather solution of the RMBequations can be used to obtain the generation of McCall-Hahn $2 \pi$-pulses. In [6] it was shown that the RMB breather is a real solution in the form of localised pulse with internal oscillations. Hence, this is an exact analogue of the McCall-Hahn 0 $\pi$-pulse. If the frequency of internal oscillations increases, then the envelope of RMB breather can be described by the soliton solution of the SIT equations to a high accuracy. Thus admittedly, the $2 \pi$-pulse of McCall-Hahn is the limiting case of the $0 \pi$-pulse of the RMB equations. 


\subsection{Optical solitons in fibres}

It is well known that the rate of the information transfer by means of fibre optical communication systems (FOCS) with the mode pulse-code modulation is limited mainly by the effect of dispersion of group velocities. The influence of this effect can be suppressed efficiently and ideally can be completely excluded if one uses sufficiently powerful pulses of light. Because of the non-linear effect of self-influence such pulses in the certain conditions are transformed in solitons and their propagation in FOCS does not accompany by dispersive spreading.

It is important to emphasise that soliton does not exist in real communication systems in the true sense of the word. The influence of group velocities dispersion of the high orders, optical loss and some other effects break a dynamic balance between the non-linear compression of pulse and its dispersion broadening. As a result of these effects the optical pulse suffers an envelope distortion and damping. But the distance, passed by the soliton in a fibre considerably exceeds one, which weak pulse should be passed in the linear regime of propagation. The experiments made with powerful optical pulses confirm this. We can consider the soliton as a good approach for the real non-linear pulses in a fibre under certain conditions.

The suggestion to use optical solitons for the information transfer along the fibre was made in the works [18, 19] and it was demonstrated [20, 21, 22]. Later on the equation describing the optical pulse propagation in a fibre with account of only the second-order group-velocities dispersion was received in works [23, 24] and was investigated in [25, 26]. In the soliton theory it was known as the non-linear Schrödinger (NLS) equation.

Let $q$ is the normalised slowly varying complex envelope of the optical pulse defined by the following expression

$$
E(x, y, z, t)=A_{0} q(z, t) \Psi(x, y) \exp \left[i\left(\beta_{0} z-\omega_{0} t\right)\right]
$$

where $\beta_{0}$ is the propagation constant depending on the frequency of the carrier wave $\omega_{0}, \Psi(x, y)$ is a mode function that determines the transverse distribution of the electric field over the fibre cross-section. Slowly varying envelope of optical pulse is governed by the follow equation [23, 24] (NLS equation):

$$
i \frac{\partial q}{\partial \zeta}+s \frac{\partial^{2} q}{\partial \tau^{2}}+\mu|q|^{2} q=0
$$


Here $\zeta=z / L_{D}, \tau=\left(t-z / v_{g}\right) t_{p 0}^{-1}$ are normalised independent variables of coordinate and time, accordingly, $t_{p 0}$ is a pulse duration at $z=0$ and $v_{g}$ is the group velocity of an optical pulse. The term in (12) with the second derivative with respect to $\tau$ describes the pulse dispersion broadening $(s=-1$ for the normal dispersion and $s=+1$ for the anomalous dispersion). $L_{D}$ is the dispersion length $L_{D}=4 \beta_{0} t_{p 0}^{2}\left(\left|\partial^{2} \beta / \partial \omega^{2}\right|\right)^{-1}$. The effective refractive index $n_{\text {eff }}$ is defined as $\beta(\omega)=(\omega / c) n_{\text {eff }}$. The third term in (12) is responsible for the self-action effect. Coefficient $\mu$ is equal to the ratio of the dispersion $L_{D}$ length to the Kerr length $L_{K}$, where $L_{K}=c^{2} \beta_{0}\left(2 \pi \omega_{0}^{2} A_{0}^{2}\left|\chi_{R, e f f}\right|\right)^{-1}$. Here $\chi_{R, e f f}$ is the effective non-linear susceptibility responsible for the Kerr effect.

The complete integrability of the NLS was established in the classical works [25, 26, 27]. Besides the multi-soliton solutions the NLS equations has the new type of the solutions named multiple-pole solutions 8 . These solutions correspond to the multiple points of a discrete spectrum of the Zakharov-Shabat problem in IST method. It is difficult to realise these solutions in practice because any small stir of the initial conditions will remove degeneration in the multiple points of a discrete spectrum. It means that only the exclusive initial optical pulses can be transformed into multiplepole optical solitons. Different properties of the NLS equations are described in the excellent books [29] - [33].

\subsection{Interaction of three waves}

One of the broadest classes of phenomena in non-linear optics is the transformation of the frequency of an electromagnetic radiation propagating in the non-linear medium. Harmonics generation of the fundamental wave (pump), sum-frequency and difference-frequency mixing are classified among these phenomena [34]. Under sufficiently high intensity of a pump the polarisation of a medium is not a linear function of the electric field strength of the wave. If the frequencies of an electromagnetic field are not in resonance with atomic transition frequencies, one can use a standard perturbation theory to reveal this dependency. So we can expand polarisation $\vec{P}$ in a power series of electrical field strength. The coefficients of this series are tensors of the $n$th rank $\hat{\chi}^{(n)}$, named as non-linear susceptibility, describe different processes of the electromagnetic waves interaction. The non-linear effects, described by the $n$th rank tensors of non-linear susceptibility, are often interpreted as the interaction of the $(n+1)$ waves.

Let the non-linear characteristics of a medium be described by non-linear 
susceptibility of the second order $\hat{\chi}^{(2)}$. They call it quadratic non-linear media. Let the waves with the carrier frequencies $\omega_{1}$ and $\omega_{2}$ propagate along $z$ axis. As the polarisation is the non-linear (quadratic) function of the electrical field strengths, the waves with carrier frequencies $\omega=\omega_{1} \pm \omega_{2}$, $\omega=2 \omega_{1}$ and $\omega=2 \omega_{2}$ appear in such medium. These waves, in their turn, can cause generation of new waves with the frequencies $\omega=2 \omega_{1} \pm \omega_{2}, \omega=$ $\omega_{1} \pm 2 \omega_{2}$, and so on. But in a dispersive medium all these processes are not equally efficient. There is a condition of phase matching, which selects a certain type of interaction of three waves, leaving all other unaffected. Sometimes such phase matching takes place for the waves propagating in the same direction. In this case they say about collinear parametric interaction. In this case the distance where the interaction of waves occurs can be made sufficiently long and, consequently, the effective frequency transformation will take place. On the contrary, when the phase matching is achievable only for the waves propagating in different directions, their interaction occurs only in the field of overlapping of the wave beams. Non-collinear parametric interaction is worth to draw special attention as the number of interesting results concerning the integrability of three-wave mixing equations have been found.

Let $\mathcal{E}_{1}, \mathcal{E}_{2}$ and $\mathcal{E}_{3}$ are the slowly varying envelopes of the interacting pulses. Let us consider the situation when only the collinear propagating wave with sum-frequency or difference frequency is generated. In the slowly varying envelopes and phases approximation, the system of equations describing the interaction of the three waves can be written in the following in unified form [35, 36]:

$$
\begin{aligned}
& \left(\frac{\partial}{\partial z}+\frac{1}{v_{1}} \frac{\partial}{\partial t}\right) q_{1}=i \sigma q_{2}^{*} q_{3}^{*} \exp (+i \Delta k z) \\
& \left(\frac{\partial}{\partial z}+\frac{1}{v_{2}} \frac{\partial}{\partial t}\right) q_{2}=i \sigma q_{3}^{*} q_{1}^{*} \exp (+i \Delta k z) \\
& \left(\frac{\partial}{\partial z}+\frac{1}{v_{3}} \frac{\partial}{\partial t}\right) q_{3}=-i \sigma q_{1}^{*} q_{2}^{*} \exp (+i \Delta k z)
\end{aligned}
$$

where $\sigma=\sqrt{\gamma_{1} \gamma_{2} \gamma_{3}}$, and $\gamma_{n}=4 \pi \omega_{n} \chi^{(2)}\left(\omega_{1}, \omega_{2}\right) / c n\left(\omega_{n}\right), n=1,2,3$. In these equations we used $\Delta k=k_{3}-\left(k_{1}+k_{2}\right)$, for a sum-frequency mixing process $\omega=\omega_{1}+\omega_{2}$, and $\Delta k=k_{3}-\left(k_{1}-k_{2}\right)$, for difference-frequency mixing 
$\omega=\omega_{1}-\omega_{2}$. Here $v_{n}$ are the group velocities of the corresponding wave. The effects of group-velocity dispersion are neglected. The uniformization variables $q_{1,2,3}$ related with $\mathcal{E}_{1}, \mathcal{E}_{2}$ and $\mathcal{E}_{3}$ as follows $\mathcal{E}_{1}=\sqrt{\gamma_{1}} q_{1}, \mathcal{E}_{2}=\sqrt{\gamma_{2}} q_{2}$ and $\mathcal{E}_{3}=\sqrt{\gamma_{3}} q_{3}^{*}$, , for the case of sum-frequency mixing, and $\mathcal{E}_{1}=-\sqrt{\gamma_{1}} q_{3}$, $\mathcal{E}_{1}=\sqrt{\gamma_{2}} q_{2}^{*}, \mathcal{E}_{3}=\sqrt{\gamma_{3}} q_{1}^{*}$ for the case of difference-frequency mixing (the indices at the velocities of the first and the third waves should be changed in this case).

We would like to point out that the resonance Raman scattering under condition of weak variation of the energy levels population of a medium and the scattering of optical waves by an acoustical wave can be considered from one position as the specific realisations of the three-wave interaction. In both cases the systems of equations, describing these processes, can be transformed into one universal system (13). Furthermore, one can demonstrate that under certain conditions the reduced Maxwell-Bloch equations appear here, which describe the propagation of the ultra-short pulse in a resonance medium. Due to this property the Raman scattering can be analysed in terms of IST method.

The equations for 3-wave interaction (13) permit both the infinite number of conservation laws and the Bäcklund transformation. They can be presented as the Hamiltonian equations by employing the $r$-matrix. It was shown that these equations pass the Painleve test and that there is a class of self-similar solutions, expressed in terms of Painleve transcendents P-V and P-VI.

It is remarkable that the system of equations, describing the parametric interaction of three waves, can be solved by IST method for a 3D case [37, 38], whereas the most of soliton equations are one-dimensional ones.

It should be emphasised that the 3-wave interaction gives an example of the dispersionless propagation of the non-linear waves. They often say that soliton is the result of the compensation of dispersion broadening and nonlinear compression of the wave packet. The 3-wave interaction just demonstrates the narrowness of this statement. Due to the absence of the phase and group velocities dispersion the solitons in this process do not detach from the non-soliton part of the solution (which is often named radiation). It seems very difficult to study the process analytically, so one has to confine the investigation to some particular solutions. The non-collinear second harmonic generation provides an example of a specific case of the 3-wave interaction, where an exact solution was found without employing the IST method. The solution obtained explicitly illustrates the non-separability of the soliton and 
non-soliton parts of the solution of these three wave interaction equations.

\section{New examples of the integrable systems}

\subsection{Generalisation of the self-induced transparency the- ory}

The development of the SIT theory is characterised by its going beyond the framework of two-level approximation and by the spectral composition of the USP field becoming more complex. The latter means that the resonant medium interacts with radiation containing several carrier frequencies. Besides, such factors as the direct interaction between resonant atoms, nonlinear properties of the dielectric doped with resonant atoms and polarisation of the electromagnetic field should be taken into account.

Let the optical pulse propagate along axis $z$ and the electric field strength be presented in the form $\vec{E}=\overrightarrow{\mathcal{E}}(t, z) \exp \left(-i \omega_{0} t+i k_{0} z\right)+$ c.c. The carrier frequency is in resonance with the frequency $\omega_{21}=\left(W_{2}-W_{1}\right) / \hbar$ of an atomic transition $j_{2} \rightarrow j_{1}$ between energy levels $W_{2}$ and $W_{1}$ degenerated over the projections $m$ and $l$ of the total angular momenta $j_{1}$ and $j_{2}$. In a general case the evolution of the envelope of the USP and states of the resonant medium are described by the system of equations for which the exact solution is not known in the case of arbitrary values $j_{1}$ and $j_{2}$. But the certain choice of transitions $j_{1}=0 \leftrightarrow j_{2}=1, j_{1}=1 \leftrightarrow j_{2}=1$, and $j_{1}=1 / 2 \leftrightarrow j_{2}=$ $1 / 2$ makes this system of equations exactly integrable. Its solution can be obtained by the inverse scattering transform method, as it was shown in [39, 40, 41]. Generalised reduced self-induced transparency equations (GSIT equations), for transitions $j_{1}=0 \leftrightarrow j_{2}=1, j_{1}=1 \leftrightarrow j_{2}=1$, can be represented in the homogeneous form

$$
\begin{gathered}
\frac{\partial q_{j}}{\partial \zeta}=-i \sum_{a=1,2} \beta_{a}\left\langle P_{j}^{(a)}\right\rangle \\
i\left(\frac{\partial}{\partial \tau}-i \Delta \omega t_{p 0}\right) P_{j}^{(a)}=\sum_{l} q_{l} M_{l j}^{(a)}-q_{j} N^{(a)}, \\
\frac{\partial}{\partial \tau} M_{j l}^{(a)}=-i\left(q_{l}^{*} P_{j}^{(a)}-q_{l} P_{j}^{(a) *}\right), \quad \frac{\partial}{\partial \tau} N^{(a)}=i \sum_{j}\left(q_{j}^{*} P_{j}^{(a)}-q_{j} P_{j}^{(a) *}\right) .
\end{gathered}
$$


If we consider transition $j_{1}=0 \rightarrow j_{2}=1$, then in (14) we assign $q_{j}=$ $d \mathcal{E}^{j} t_{p 0} / \hbar, \beta_{1}=1$ and $\beta_{2}=0$. For transition $j_{1}=1 \rightarrow j_{2}=0$ we have $q_{j}=d \mathcal{E}^{j} t_{p 0} / \hbar, \beta_{1}=0$ and $\beta_{2}=1$. Finally, for transition $j_{1}=1 \rightarrow j_{2}=1$ we have $q_{j}=j d \mathcal{E}^{j} t_{p 0} / \hbar \sqrt{2}, \beta_{1}=\beta_{2}=1 / 2$. Everywhere here the sub-index and upper index take the values $j= \pm 1$. The slowly varying envelopes of matrix elements of the density matrix $\hat{\rho}$ are determined as $P_{j}^{(1)}=\left\langle j_{2}, 0|\hat{\rho}| j_{1}, j\right\rangle$, $P_{j}^{(2)}=\left\langle j_{2},-j|\hat{\rho}| j_{1}, 0\right\rangle, N^{(1)}=\left\langle j_{2}, 0|\hat{\rho}| j_{2}, 0\right\rangle, N^{(2)}=-\left\langle j_{1}, 0|\hat{\rho}| j_{1}, 0\right\rangle, M_{j l}^{(1)}=$ $\left\langle j_{2}, j|\hat{\rho}| j_{1}, l\right\rangle, M_{j l}^{(2)}=\left\langle j_{2},-l|\hat{\rho}| j_{1},-j\right\rangle$. As we are well aware, GSIT equations are the zero-curvature condition in the vector expansion IST method for the AKNS hierarchy. The spectral problem with such a sort of IST was first reported by Manakov 42 to describe the self- focusing of the polarised light beams. The expressions for $N$-soliton solutions, breathers and Bäcklund transformation have been found in [43].

There are versions of the GSIT equations describing USP propagation in three-level medium. In the simplest case of this model the resonance levels have $V$ and $\Lambda$ configurations. It was determined [44, 45] that, if the oscillator forces for every transition in a $V$ or $\Lambda$ configuration are equal, then a two-frequency pulse (characterised by two different frequencies of the carrier wave) is able to propagate in such a medium without the envelope distortion. An ultra-short pulse of the kind was called simulton [46]. The simultons are single-soliton solutions of the above GSIT equations. At the same time solutions occur which are responsible for the propagation and collisions of simultons. The oscillating simultons (colour breathers) are the two-frequency generalisations of $0 \pi$-pulse by McCall-Hahn. It is worth noting at this point that a simulton is generally unstable with respect to transformation into onefrequency $2 \pi$-pulse and may remain as two-frequency pulse only for a special choice of resonance level populations.

It should be mentioned that consideration of the polarised USP propagation ion three-level medium leads to matrix variants of the GSIT-equations. For more details, see Ref. [47, 48]. The matrix expansion IST method for the AKNS hierarchy (or matrix Manakov spectral problem) there occurs.

\subsection{Femtosecond optical solitons in fibres}

In order to describe the non-linear phenomena associated with femtosecond optical solitons in non-linear fibres, the higher-order non-linear Schrödinger (HNLS) equation has been proposed [49, 50] (see also [51]). Consider the 
following generalisation of the equation (12)

$$
i \frac{\partial q}{\partial \zeta}+s \frac{\partial^{2} q}{\partial \tau^{2}}+\mu|q|^{2} q+i\left(\eta_{3} \frac{\partial^{3} q}{\partial \tau^{3}}+\mu_{2}|q|^{2} \frac{\partial q}{\partial \tau}+\mu_{3} q \frac{\partial|q|^{2}}{\partial \tau}\right)=0 .
$$

The parameter $\eta_{3}$ corresponds to the third-order group-velocity dispersion, parameters $\mu_{2}$ and $\mu_{3}$ represent the two inertial contributions to the nonlinear polarisation, i.e., Raman self- scattering and self-steeping formation. If $\eta_{3}=0, \mu_{2}=\mu_{3}=1$, and $\mu=0$, then the HNLS (15) reduced into derivative non-linear Schrödinger (DNLS) equation

$$
i \frac{\partial q}{\partial \zeta}+\frac{\partial}{\partial \tau}\left(s \frac{\partial q}{\partial \tau}+i|q|^{2} q\right)=0 .
$$

This equation is completely integrable one [52]. There are soliton and multisoliton solutions, which can be obtained in the framework of IST method. Notice that the condition $\mu=0$ is not essential for reduction of the HNLS into the integrable equation, i.e., resulting modified DNLS equation is completely integrable too.

If $\eta_{3}=1, \mu_{2}= \pm 6, \mu_{3}=0$, then the equation (15) is reduced to the Hirota equations 53.,

$$
i \frac{\partial q}{\partial \zeta}+s \frac{\partial^{2} q}{\partial \tau^{2}}+\mu|q|^{2} q+i \frac{\partial^{3} q}{\partial \tau^{3}} \pm 6 i|q|^{2} \frac{\partial q}{\partial \tau}=0
$$

which represents other example of completely integrable equation.

In general, equation (15) may not be completely integrable. However, if we suppose that $\eta_{3}=\varepsilon, \mu_{2}=6 \varepsilon, \mu_{3}=3 \varepsilon$, and $\mu=2 s$, then the HNLS equation can be reduced to the other one - Sasa-Satsuma equation. In this case in terms of new variables $\xi=\tau-s^{2} \zeta / 3 \varepsilon, q(\tau, \zeta)=u(\xi, \zeta) \exp (i s \tau / 3 \varepsilon+$ $2 i s^{3} \zeta / 27 \varepsilon^{2}$ ) the equation (15) takes the form

$$
\frac{\partial u}{\partial \zeta}+\varepsilon\left(\frac{\partial^{3} u}{\partial \xi^{3}}+6|u|^{2} \frac{\partial u}{\partial \xi}+3 u \frac{\partial|u|^{2}}{\partial \xi}\right)=0
$$

The equation (18) has been considered in ( 54$]$, where it was shown that this equation could be solvable by means of IST method. 


\subsection{Vector optical solitons}

In the general case the vector soliton is the soliton solution of the non-linear system of the evolution equations, which one can be presented as the onedimension array. For example, the vector soliton of the NLS equation is the solution of the following vector equation

$$
i \frac{\partial \vec{q}}{\partial \zeta}+\frac{1}{2} \frac{\partial^{2} \vec{q}}{\partial \tau^{2}}+\left(\vec{q} \vec{q}^{*}\right) \vec{q}=0
$$

where $\vec{q}=\left\{q_{1}, q_{2}, \ldots, q_{M}\right\}$. Hereafter, this equation will referred to as the $v$-NLS equation. The vector index of this soliton can be resulted from the different physical origin.

We have only one example where the v-NLS equation is the completely integrable one. This equation is embedded into AKNS hierarchy that allows to exploit the IST method to find the soliton solution. The suitable spectral problem was found by Manakov 42].

As the simplest vector generation of the DNLS equation one can write the standard form of the $v$-DNLS equation

$$
i \frac{\partial \vec{q}}{\partial \zeta}+\frac{\partial}{\partial \tau}\left(\frac{\partial \vec{q}}{\partial \tau}-i \varepsilon\left(\vec{q} \vec{q}^{*}\right) \vec{q}\right)=0
$$

Other example of the vector non-linear waves arises under consideration ultra-short optical pulse propagation in birefringent fibres with higher order effects like the third order dispersion of group velocities, Kerr dispersion, and stimulated Raman scattering. There is two-component generalisation of the Sasa-Satsuma equation (18) [55]:

$$
\frac{\partial \vec{q}}{\partial \zeta}+\varepsilon\left(\frac{\partial^{3} \vec{q}}{\partial \xi^{3}}+6\left(\vec{q} \vec{q}^{*}\right) \frac{\partial \vec{q}}{\partial \xi}+3 \vec{q} \frac{\partial\left(\vec{q} \vec{q}^{*}\right)}{\partial \xi}\right)=0 .
$$

In [55] the three-component generalisation of this equation was considered too. It was shown that these equations have the zero-curvature representation and can be solved by IST method. However, the exact soliton solutions more simply to obtain using the Darboux- Bäcklund transformation. 


\subsection{Extremely short pulse propagation in non-resonant medium}

The recent progress in the field of generation of femtosecond pulses has made it necessary to revise theoretical models of their propagation in a non-linear dispersive medium. It is interesting to find a method to describe an USP evolution without the use of slowly varying envelope approximation. The simplest way to do this is to combine the wave equation for electromagnetic field with the equations specifying the changes in the state of the medium.

In the case of resonant medium we have the resonance transition frequency as a scale parameter for time. When pulse duration $t_{p}$ obeys the inequality $t_{p} \omega_{a} \gg 1$, the slowly varying envelope approximation is adequate to describe the pulse propagation. On the contrary, if $t_{p} \omega_{a} \ll 1$ we can use at least the unidirectional propagation approximation. The ratio $\varepsilon=\omega_{R} / \omega_{a}$, where $\omega_{R}$ is the Rabi frequency (i.e., $\omega_{R}=d \max |E| / \hbar$ ), provides a new parameter. Let the USP amplitude (i.e., $E$ ) be of such magnitude that the Rabi frequency $\omega_{R}$ is small compared with the minimum of atomic transition frequency. That means $\varepsilon=\omega_{R} / \omega_{a}$ is a small parameter. Then we can attempt to solve the Bloch equations (4) approximately and thus obtain an approximate equations of the USP electric field strength without the assumption of slowly varying envelope [56]. The Maxwell equations under unidirectional wave approximation can be reduced into

$$
\frac{\partial E}{\partial z}+\frac{1}{c} \frac{\partial E}{\partial t}=-\frac{2 \pi n_{A} d}{c}\left\langle\frac{\partial P_{1}}{\partial t}\right\rangle
$$

where polarisation of the ensemble of two-level atoms at the third order of $\varepsilon$ is

$$
\left\langle P_{1}\right\rangle=\left\langle\frac{2 d}{\hbar \omega_{a}}\right\rangle E-\left\langle\frac{2 d}{\hbar \omega_{a}^{3}}\right\rangle \frac{\partial^{2} E}{\partial t^{2}}-\left\langle\frac{4|d|^{2} d}{\hbar^{3} \omega_{a}^{3}}\right\rangle E^{3} .
$$

Substitution of the expression for polarisation into equation (22) in terms of new variables $\tau=|b| z, \zeta=t-z / V, u(\tau, \zeta)=-(a / 6 b)^{1 / 2} E(z, t)$ yields the modified Korteweg-de Vries equation ( $\mathrm{mKdV})$

$$
\frac{\partial u}{\partial \tau}+6 u^{2} \frac{\partial u}{\partial \zeta}+\frac{\partial^{3} u}{\partial \zeta^{3}}=0
$$

Here parameters $a=\left\langle 24 \pi n_{A}|d|^{4} / c \hbar^{3} \omega_{a}\right\rangle, b=\left\langle 4 \pi n_{A}|d|^{2} / c \hbar^{3} \omega_{a}\right\rangle$ were in- 
troduced. The expression $V^{-1}=c^{-1}\left[1+\left\langle 4 \pi n_{A}|d|^{2} / \hbar \omega_{a}\right\rangle\right]$ defines the renormalised velocity of the USP propagation. As it is known [57] this equation is a completely integrable, and its solutions can be found by the IST method [31, 32, 33]. There are another non-linear equations describing extremely short pulse propagation [58, however, they have not soliton solutions.

\subsection{SIT in a Kerr-type non-linear medium}

There are intensive investigations of the non-linear pulse propagation in the optical fibres [29, 30]. However, practically all materials used for fibre fabrication contain impurities that contribute to the absorption spectrum of the fibres. The losses due to the resonant absorption decrease if the frequency of the carrier wave is located within the window of transparency of glass fibre. Another means to decrease losses is to make pulse duration shorter than the characteristic relaxation times of the resonant states or in other words to make optical pulses ultra-short. In this case the well-known self-induced transparency phenomenon can be expected to arise.

It is known that the non-linear Schrödinger equation, which is used to describe optical solitons in non-linear monomode optical fibre, is completely integrable [31, 32, 33. The reduced Maxwell-Bloch equations or their generalisations considered hereafter, as RMB-equations are completely integrable too. The IST method for both the NLS and the RMB-equations enabling to solve some non-linear evolution equations is based on the same spectral problem. The model of the USP propagation in a Kerr-type non-linear medium doped by resonant impurity atoms incorporates both of these systems. But there are no reasons for the resulting system of equations to possess a complete integrability.

The evolution of the USP propagating in a non-linear monomode optical fibre in $z$ - direction is described by the equations, which generalise the Maxwell-Bloch equations [59]. We could name them the non-linear Schrödinger and Bloch equations.

$$
\begin{gathered}
i \frac{\partial q}{\partial \zeta}+s \frac{\partial^{2} q}{\partial \tau^{2}}+\mu|q|^{2} q+a\langle p\rangle=0 \\
\frac{\partial p}{\partial \tau}=i \delta p+2 i f R_{3}, \quad \frac{\partial R_{3}}{\partial \tau}=i f\left(q^{*} p-q p^{*}\right),
\end{gathered}
$$


where $q$ is the normalised slowly varying complex envelope of the USP defined by the following expression

$$
E(x, y, z, t)=A_{0} q(z, t) \Psi(x, y) \exp \left[i\left(\beta_{0} z-\omega_{0} t\right)\right]
$$

$\Psi(x, y)$ is a mode function that determines the transverse distribution of the electric field over the fibre cross-section. Here $\zeta=z / L_{D}, \tau=\left(t-z / v_{g}\right) t_{p 0}^{-1}$, are normalised independent variables of co-ordinate and time, accordingly, $t_{p 0}$ is a pulse duration at $z=0$ and $v_{g}$ is the USP propagation group velocity. The interaction of the radiation with the resonant impurities is characterised by the dimensionless constant $f=\bar{d} A_{0} t_{p 0} / \hbar$, where $\bar{d}$ is an effective matrix element of the dipole transition between the resonant states. The coefficient $a$ is expressed in terms of the dispersion length $L_{D}$ and the resonant absorption length $L_{a}$ [48 as $a=L_{D} L_{a}^{-1} f^{-1}$, where $L_{a}=c \hbar n_{\text {eff }}\left(2 \pi \omega_{0} n_{A} \bar{d}^{2} t_{p 0}\right)^{-1}$.

Now let us consider ultra-short pulses propagation in a Kerr-type dispersive medium when the transition between energy levels of the impurity atoms are degenerated over the orientations of the total angular momentum $j_{1}$ and $j_{2}$. The same system of equations appears when fibre contains threelevel impurity atoms, so that formally we could speak about optical vector solitons in a general case. Let the electric field strength write as

$$
E^{(j)}(x, y, z, t)=A_{0} q_{j}(z, t) \Psi(x, y) \exp \left[i\left(\beta_{0} z-\omega_{0} t\right)\right] .
$$

The equations for the normalised envelope and the variables of the atomic resonant system can be written in a unified form as it was done above

$$
\begin{gathered}
i \frac{\partial q_{j}}{\partial \zeta}+s \frac{\partial^{2} q_{j}}{\partial \tau^{2}}+\mu|\vec{q}|^{2} q_{j}-a\left\langle P_{j}\right\rangle=0 \\
\frac{\partial P_{j}^{(a)}}{\partial \tau}-i \delta P_{j}^{(a)}=-i f\left(\sum_{l} q_{l} M_{l j}^{(a)}-q_{j} N^{(a)}\right), \\
\frac{\partial}{\partial \tau} M_{j l}^{(a)}=-i f\left(q_{l}^{*} P_{j}^{(a)}-q_{l} P_{j}^{(a) *}\right), \quad \frac{\partial}{\partial \tau} N^{(a)}=i f \sum_{j}\left(q_{j}^{*} P_{j}^{(a)}-q_{j} P_{j}^{(a) *}\right)
\end{gathered}
$$

where $j$ and $l$ mark the spherical components of the vectors, and $P_{j}=$ $\sum_{a} \beta_{a} P_{j}^{(a)}$. The variables into the Bloch equations in the system (27) have been determined above. 
It has been found [48] that both system of equations (25-26) and (27) are integrable ones only under condition $L_{D} L_{K}^{-1}=2 f^{2}$. This condition implies that the soliton of the self-induced transparency should simultaneously be also a soliton of the NLS equation. To put this another way, the amplitude and duration of the $2 \pi$-pulse should precisely be of such values that the corresponding self-action (due to the high-frequency Kerr effect) would lead to complete compensation of the dispersion broadening of the USP. Thereby, the existence of the optical solitons in a fibre doped with resonant impurities is restricted.

Yet another example of integrable model describing USP propagation in fibre was proposed in 60. There the NLS equation (25) has been replaced by the Hirota-like equation:

$$
i \frac{\partial q}{\partial \zeta}+\frac{1}{2} \frac{\partial^{2} q}{\partial \tau^{2}}+|q|^{2} q+i \varepsilon\left(\frac{\partial^{3} q}{\partial \tau^{3}}+3|q|^{2} \frac{\partial q}{\partial \tau}+\frac{3}{2} q \frac{\partial|q|^{2}}{\partial \tau}\right)+a\langle p\rangle=0
$$

The resulting system of equation admits the Painleve property for certain relation between the physical parameters involved in the model. Also the zero-curvature representation of this system was explicitly found.

\section{Conclusion}

In conclusion it will be considered some problems related with an integrable models and developments of the ideas mentioned above.

In the traditional scheme of the IST method the spectral parameter of the auxiliary linear problem is considered as a constant. The generation of the IST method, where the spectral parameter depends on time and space co-ordinate was proposed in 61]. Non-linear equations arising in this approach include the explicit dependence on co-ordinates. In framework of this method the generation of the Maxwell-Bloch equations was constructed. This new system describes evolution of the USP in two-level medium wherein the pumping of the excited states of atoms operated continuously.

Propagation of the USP in a long two-level amplifier was considered in 62, 63. The IST method was applied to the SIT equations in order to obtain its uniform asymptotic solution at long distance. It was shown that the amplified pulse is always of a quasi self-similar nature. In the neighbourhood 
of the wave front USP is described by the Painleve equation, whereas far from the front the solution goes into the rapidly oscillating self-similar regime.

The SIT equations has been employed 64 to describe the phenomenon of superfluorescence (i.e., short pulse generation from initial fluctuations of polarisation in a high inverted of the population in two-level medium). Contrary to the self-induced transparency, the superfluorescence pulse is generating from an unstable state and IST method is to be re-formulated for boundary problem at the half $t$-axis. It was done in 64.

Progress in laser physics has made possible the generation of intense ultrashort pulses to produce the multi-photon processes. The simplest one develops when the resonant matter interacts with a pair of USPs of different carrier frequencies $\omega_{1}$ and $\omega_{2}$ so that $\omega_{1} \pm \omega_{2}$ coincides with a transition frequency $\omega_{21}$. The non-linear processes are two-photon absorption and Raman scattering, respectively. The coherent pulse propagation takes place under both resonance conditions when the pulse duration is much less then relaxation times. This process is similar to SIT and it was referred to as two-photon self-induced transparency (TPSIT). This phenomenon can be described in framework of the generalised Maxwell-Bloch equations [65, 66]. These equations were converted into new system of equation for new variables, which are quadratic functions of initial slowly varying envelopes of the electromagnetic fields [66, 67]. As was shown by Kaup and Steudel [67, 68] under certain conditions these equations can be solved by using modified IST method.

It is interesting that both McCall-Hahn equations in the sharp-line limit and exact resonance and GSIT equations can be mapped into the model of principal chiral fields. The Kaup-Steudel equations are related with the same model [69]. Recently the authors of [70] have investigated the GSIT equations and found the hidden non-Abelian group structure of these equations in the case of multi-level resonant medium. They have discovered that a non-degenerate two-level system of self-induced transparency is associated with symmetric space $G / H=S U(2) / U(1)$ while three-level $V$ - or $\Lambda$-systems are associated with $G / H=S U(3) / U(2)$. The same symmetric space is associated with the degenerate two-level system of SIT in the case of transitions $j_{1}=1 \rightarrow j_{2}=0$ and $j_{1}=0 \rightarrow j_{2}=1$. When one considers the transition $j_{1}=1 / 2 \rightarrow j_{2}=1 / 2$, the GSIT equations are associated with $G / H=(S U(3) / U(2))^{2}$. There are many complex aspects related to the degeneration of the energy levels in a three-level system of SIT. For instance, the transitions between states $j_{a}=j_{c}=0, j_{b}=1$ ( or $j_{a}=j_{c}=1, j_{b}=0$ ) are associated with the symmetric space $G / H=S U(4) / S(U(2) \times U(2))$ 
(accordingly $G / H=S U(5) / U(4))$.

The degenerate four-wave interaction consisting of two counter-propagating pulses in a cubic non-linear medium, with arbitrary polarisations which can vary through each pulse has been considered in [71, 72]. The group velocity dispersion was neglected that results in the intensity envelopes propagating unchanged from their initial forms. However, the polarisation evolves according to the non-linear interaction and it is found it can exhibit soliton behaviour. The problem is best approached if one introduce the following vectors (i.e., Stokes vectors)

$$
\mathbf{S}^{( \pm)}=\left\{q_{x}^{( \pm) *} q_{y}^{( \pm)}+\text {c.c. }, \quad i q_{x}^{( \pm) *} q_{y}^{( \pm)}+\text {c.c. }, \quad\left|q_{x}^{( \pm)}\right|^{2}-\left|q_{y}^{( \pm)}\right|^{2}\right\}
$$

where $q_{x, y}^{( \pm)}$are the transverse normalised slowly varying envelopes of the pulses propagating in the $+\hat{\mathbf{z}}$ and $-\hat{\mathbf{z}}$ directions, respectively. By using the cone co-ordinate $2 x_{ \pm}=\mp(z \pm c t)$, the evolution equations for can be rewritten as the equation of motion for chiral field on the group $O(3)$ :

$$
\partial_{+} \mathbf{S}^{(+)}=\mathbf{S}^{(+)} \times \hat{J} \mathbf{S}^{(-)}, \quad \partial_{-} \mathbf{S}^{(-)}=-\mathbf{S}^{(-)} \times \hat{J} \mathbf{S}^{(+)}
$$

where $\hat{J}$ diagonal matrix is defined by constants of the model. These equations are integrable by IST method [73, 74]. It should be pointed that in the anisotropic case (i.e., matrix elements of are different) the equations (29) describe the domain wall (DW) in the configuration of the Stokes vectors. As far as we know it is the first example of domain structure in non-linear optics, where DW's represent space regions with different stable polarisation states of the interacting optical waves. The Hamiltonian achieves the minimum on these optical domains, and DW's are the regions of polarisation switching. Recently, study of the DW's was considered in systems of coupled NLS equations governing propagation of light in non-linear fibres [75]. An important point is that group-velocity dispersion was taken into account. The two types of the DW were found: the one between different elliptic polarisations in the bimodal fibre and a dark soliton in one core of the dual-core coupler. However, both the model considered in [75] and models quoted there are not integrable ones.

\section{Acknowledgment}

I am grateful to Prof. S.O. Elyutin and Dr. A.M. Basharov for enlightening discussions. Also financial support from INTAS (grant No: 96-0339) is 
acknowledged. 


\section{References}

[1] S.L. McCall and E.L. Hahn, Phys.Rev.Letts. 18, 908 (1967)

[2] S.L. McCall and E.L. Hahn, Phys.Rev. 183, 457 (1969)

[3] L. Allen and J.H. Eberly, Optical Resonance and Two-Level Atoms (Wiley, New York, 1975).

[4] R.F. Slusher, Progr.Optics, 12, 53 (1974)

[5] G.L. Lamb, Jr., Rev.Mod Phys. 43, 99 (1971)

[6] J.L. Eilbeck, J.D. Gibbon, P.J. Caudrey and R.K. Bullough, J.Phys. A 6, 1337 (1973).

[7] G.L. Lamb, Jr. Phys.Rev. A 9, 422 (1974).

[8] J.D. Gibbon, P.J. Caudrey, R.K. Bullough and J.L. Eilbeck, Letts.Nuovo Cimento 8, 755 (1973).

[9] L.A. Takhtajan, Sov.Phys. JETP 39, 228 (1974)

[10] M.J. Ablowitz, D.J. Kaup, A.C. Newell and H. Segur, J.Math.Phys. $\mathbf{1 5 , 1 8 5 2 ( 1 9 7 4 )}$

[11] D.J. Kaup, Phys.Rev. A 16, 240 (1965).

[12] H.A. Haus, Rev.Mod.Phys. 51, 331 (1979).

[13] J.D. Gibbon and J.L. Eilbeck, J.Phys. A 5, L122 (1972).

[14] P.J. Caudrey, J.D. Gibbon., J.L. Eilbeck and R.K. Bullough, Phys. Rev. Letts. 30, 237 (1973)

[15] P.J. Caudrey, J.L. Eilbeck, J.D. Gibbon and R.K. Bullough, J.Phys. A 6, L53 (1973)

[16] R.K. Bullough, P.M. Jack, P.W. Kitchenside and R. Saunders, Phys.Scr. 20, 364 (1979).

[17] J.L. Eilbeck and R.K. Bullough, J.Phys. A 5, 820 (1972).

[18] A. Hasegawa and F. Tappert, Appl.Phys.Lett. 23, 142 (1973). 
[19] A. Hasegawa and F. Tappert, Appl.Phys.Lett. 23, 171 (1973).

[20] D.M. Bloom, L.F. Mollenauer, Ch. Lin, N. Taylor and A.M. Del Gaudio, Opt.Lett. 4, 297 (1979).

[21] L.F. Mollenauer, R.H. Stolen and J.P. Gordon, Phys.Rev.Letts. 45, 1095 (1980).

[22] L.F. Mollenauer, R.H. Stolen and M.N. Islam, Opt.Lett. 10, 229 (1985).

[23] M. Jain and N. Tzoar, J.Appl.Phys. 49, 4649 (1978).

[24] B. Bendow, P.D. Gianino, N. Tzoar and M. Jain, J.Opt.Soc.Amer. 70, 539 (1980).

[25] V.E. Zakharov and A.B. Schabat, Sov.Phys. JETP 34, 62 (1972).

[26] V.E. Zakharov and S.V. Manakov, Teor.Mat.Fiz. 19, 332 (1974) (in Russian).

[27] J. Satsuma and N. Yajima, Progr. Theor. Phys. Suppl. N.55, 284 (1974).

[28] E. Olmedilla, Physica D 25, 330 (1987).

[29] A. Hasegawe, Optical Solitons in Fibers (Springer-Verlag, Berlin, 1990).

[30] G.P. Agrawal, Nonlinear Fiber Optics (Academic Press, Inc., Boston, San Diego, New York, London, Sydney, Tokyo, Toronto, 1989).

[31] M.J. Ablowitz and H. Segur, Solitons and the Inverse Scattering Transform (SIAM, Philadelphia, 1981).

[32] L.A. Takhtajan and L.D. Faddeev, Hamiltonian approach to theory of solitons (Nauka, Moscow, 1986).

[33] R.K. Dodd, J.C. Eilbeck, J.D. Gibbon and H.C. Morris, Solitons and Nonlinear Waves (Academic Press, New York, 1982).

[34] Y.R. Shen, The principles of non-linear optics (John Wiley \& Sons, New York, Chicester, Brisbane, Toronto, Singapore, 1984).

[35] V.E. Zakharov and S.V. Manakov, Zh.Eksp.Teor.Fiz. 69, 1654 (1975). 
[36] D.J. Kaup, Stud. Appl. Math. 55, 9 (1976).

[37] D.J. Kaup, Physica D 3, 374 (1981).

[38] D.J. Kaup, Stud.Appl.Math. 62, 75 (1980)

[39] A.I. Maimistov, Opt.Spectrosk. 57, 564 (1984) (in Russian).

[40] A.M. Basharov and A.I. Maimistov, Sov.Phys. - JETP 69, 913 (1984).

[41] A.M. Basharov, A.I. Maimistov and Yu.M. Sklyarov, Opt.Spectrosc. 62, 418 (1987).

[42] S.V. Manakov, Sov.Phys. JETP 38, 248 (1974).

[43] H. Steudel, J.Mod.Opt. 35, 693 (1988).

[44] M.J. Konopnicki and J.H. Ebedly, Phys.Rev. A 24, 2567 (1981).

[45] A. Kujawski, Opt.Commun. 43, 375 (1982).

[46] M.J. Konopnicki, P.D. Drummond and J.H. Eberly, Appl.Phys. B 28, 103 (1982).

[47] A.M. Basharov and A.I. Maimistov, Sov.Phys. JETP 67, 2426 (1988).

[48] A.I. Maimistov, A.M. Basharov, S.O. Elyutin and Yu.M. Sklyarov, Phys.Rept. C 191, 1 (1990).

[49] Y. Kodama, J.Stat.Phys. 39, 597 (1985).

[50] Y. Kodama, A. Hasegawa, IEEE J.Quant.Electron. QE-23, 510 (1987).

[51] M.J. Potasek, J.Appl.Phys. 65, 941 (1989).

[52] D.J. Kaup and A.C. Newell, J.Math.Phys. 19, 798 (1978).

[53] R. Hirota, J.Math.Phys. 14, 805 (1973).

[54] N. Sasa and J. Satsuma, J.Phys.Soc.Japan 60, 409 (1991).

[55] K. Nakkeeran, K. Porsezian, P. Shanmugha Sundaram and A. Mahalingam, Phys.Rev.Lett. 80, 1425 (1998). 
[56] A.I. Maimistov, Opt.Spectrosc. 76, 569 (1994).

[57] M. Wadati, J.Phys.Soc.Japan 34, 1289 (1973).

[58] A.I. Maimistov, Quantum Electronics 30, 287 (2000).

[59] A.I. Maimistov and E.A. Manykin, Sov.Phys. JETP 58, 685 (1983).

[60] K. Nakkeeran and K. Porsezian, J.Phys. A. 28, 3817 (1995).

[61] S.P. Burtsev, V.E. Zakharov and A.V. Mikhailov, Teor.Math.Fiz. 70, 323 (1987).

[62] S.V. Manakov, Zh.Eksp.Teor.Fiz. 83, 68 (1982).

[63] S.V. Manakov and V.Yu. Novokshenov, Teor.Math.Fiz. 69, 40 (1986).

[64] I.R. Gabitov, V.E. Zakharov and A.V. Mikhailov, Teor.Math.Fiz. 63, 11 (1985).

[65] E.M. Belenov and I.A. Poluektov, Sov.Phys. JETP 29, 754 (1969).

[66] H. Steudel, Ann.Phys.(DDR) 34, 188 (1977).

[67] H. Steudel, Physica D 6, 155 (1983).

[68] D.J. Kaup, Physica D 6, 143 (1983).

[69] A.I. Maimistov, Phys.Lett. A 144, 11 (1990).

[70] Q.-H. Park and H.J. Shin, Phys.Rev. A 57, 4621 (1998).

[71] M.S.Tratnik and J.E. Sipe, Phys.Rev.Lett. 58, 1104 (1987).

[72] V.E. Zakharov and A.V. Mikhailov, Pis'ma v Zh.Eksp.Teor.Fiz. 45, 279 (1987).

[73] I.V. Cherednik, Teor.Math.Fiz. 47, 537 (1981).

[74] V.E. Zakharov and A.V. Mikhailov, Zh.Eksp.Teor.Fiz. 74, 1953 (1978).

[75] B.A. Malomed, Phys.Rev. E 50, 1565 (1994). 